\title{
Single Nucleotide Polymorphisms as Biomarkers for Drug Response and Toxicity in the Management of Colorectal Cancer
}

\author{
Ashok Varma, Mathaiyan Jayanthi ${ }^{*}$ and Deepak Gopal Shewade \\ Jawaharlal Institute of Post Graduate Medical Education and Research, Pondicherry, India
}

*Corresponding author: Mathaiyan Jayanthi, Jawaharlal Institute of Post Graduate Medical Education and Research, Pondicherry, India, Tel: 9442395291; E-mail: mailjayanthi@gmail.com

Received date: April 16, 2018; Accepted date: May 07, 2018; Published date: May 17, 2018

Copyright: (C) 2018 Varma A, et al. This is an open-access article distributed under the terms of the Creative Commons Attribution License, which permits unrestricted use, distribution, and reproduction in any medium, provided the original author and source are credited.

\begin{abstract}
The current methods of cancer treatment don't focus on the influence of interpatient genetic variations on the treatment outcomes. Genetic variations in the genes which are involved with drug metabolism and targets can affect treatment response and toxicity. In the recent time, many advances have been made to detect such candidate gene variations and use them as biomarkers in predicting the treatment outcomes in colorectal cancer (CRC). CRC remains one of the common causes of worldwide morbidity and mortality. In the last decade, many drugs have been approved including targeted therapies for the management of CRC which lead to improvement in the median survival and overall survival rate. However, there are significant variations in the response rate and toxicity among CRC patients with the current anti-cancer drugs. Interindividual response variations to chemotherapy can be influenced by several factors among them the genetic factor plays a key role. This emphasizes the need for the new genetic biomarkers which enable the selection of optimal drugs to benefit the patient care. This review focuses on single nucleotide polymorphisms which are known to affect the outcome of anticancer drugs used in the management of colorectal cancer.
\end{abstract}

Keywords: Nucleotide; Biomarkers; Drug; Toxicity; Colorectal cancer

\section{Introduction}

Colorectal cancer (CRC) also known as bowel cancer, often begin as a small polyp on the inner wall of the colon or rectum. Polyps are benign and adenomatous in nature. Few of these polyps become cancerous over time [1]. Early finding and removing of these polyps can prevent development of CRC.

CRC is the third most commonly diagnosed cancer and the fourth leading cause of cancer-related deaths worldwide [2]. Incidence rates of CRC are similar in both the sexes, with a slight male predominance. As per globacon report 2012, CRC is the third most commonly seen cancer in men ( $10 \%$ of the total cancers) and second most in women
(9.2\% of total cancer). In India as per the Indian Council of Medical Research (ICMR) consensus document on management of colorectal cancer released in 2014, the annual incidence rates (AARs) for colon and rectal cancer in men and women are 4.4 and 3.9 per 100000 respectively [3].

Survival rate with CRC is highly dependent upon the disease stage at the time of diagnosis. In Asian countries, the overall cure rate has not been improved in spite of several improvements in CRC management. The five-year survival rate remains $60 \%$ in Asian countries. The highest survival rates were found in China (60.8\%), the lowest rate was reported in India (31.2\%). In the United States, the 5year survival rate of CRC is of $64 \%$. The failure to diagnose in early stages due to lack of regular screening is the main reason for low survival rates in Asian countries (Table 1) [4].

\begin{tabular}{|l|l|l|}
\hline Drug & Metabolism & Drug targets \\
\hline 5-flurouracil \& Capecitabine & Dihydropyrimidine dehydrogenase & Thymidylate synthase \\
\hline Oxaliplatin & Glutathione S transferase & DNA adducts \\
\hline Irinotecan & UDP-glucuronosyl transferase & Topoisomerase \\
\hline Cetuximab & Reticuloendothelial system & Epidermal growth factor receptor \\
\hline Panitumumab & Reticuloendothelial system & Epidermal growth factor receptor \\
\hline Bevacizumab & Reticuloendothelial system & Vascular endothelial growth factor A \\
\hline
\end{tabular}

Table 1: The table shows the current drugs used in CRC management, Respective metabolizing pathways and Drug targets.

The efficacy of drugs used in the management of CRC is often limited by significant interindividual variation. Such variations are often linked to single nucleotide polymorphisms either in the drugmetabolizing enzymes, DNA repair enzymes or in drug targets [5]. 
Single nucleotide polymorphisms are single base mutation in DNA and often referred as SNP's. SNP's are the most common type of genetic variations among people. The prior genetic screening for SNP's in patients will give an insight about better responders and patients who may likely to develop toxicity with chemotherapy, so that personalized therapy can be planned based on their genetic profile in order to maximize the benefits.

\section{Single Nucleotide Polymorphism's as Predictive Markers for Drug Response and Toxicity in CRC}

\section{Drug metabolizing enzymes}

Dihydropyrimidine dehydrogenase (DPD): DPD is a rate limiting metabolizing enzyme for 5 -flurouracil and its prodrug capecitabine. The expression levels of DPD found to play key role in conferring the efficacy. Overexpression of DPD enzyme is often related to drug resistance [6].

A meta-analysis reported DPYD gene to be highly polymorphic in different ethnic population. $\mathrm{G}>\mathrm{A}$ single nucleotide polymorphism at the 5 '-splice sequence of exon $14\left(\mathrm{DPYD}^{\star} 2 \mathrm{~A}\right)$ is the most commonly reported variation of DPYD gene which leads to the formation of a truncated non-functional protein [7]. FDA marketing label of 5-FU and capecitabine clearly warns that patients who are with homozygous and heterozygous mutation in the DPYD gene results in complete or partial absence of DPD activity and are at increased risk for the early onset toxicity and severe life-threatening or fatal adverse drug reactions like mucositis, hand-foot syndrome (HFS), diarrhoea, neutropenia and neurotoxicity [8]. HFS is also called palmar-plantar erythrodysesthesia characterized by severe skin changes like peeling, blisters, bleeding, and severe pain [9]. The major limitation of this polymorphism of being used as a predictive marker for toxicity is its lower minor allele frequency in different ethnic groups and only about $50 \%$ DPYD ${ }^{\star} 2$ A allele carriers actually develop severe toxicities with 5$\mathrm{FU}[10]$.

Recent studies found that presence of a candidate haplotype, from a combination of three SNP's (rs 1801160, rs 1801265, rs 2297595) of DPYD gene can lead to the development of severe toxicities with 5-FU and capecitabine treatment in CRC patients, validating this haplotype as toxicity predictor parallel to DPYD ${ }^{\star} 2 \mathrm{~A}[11]$.

Methylenetetrahydrofolate reductase: Methylenetetrahydrofolate reductase (MTHFR) plays an indirect role in increasing the efficacy of 5-FU and capecitabine. MTHFR catalyzes the conversion of 5, 10 methylenetetrahydrofolate (5,10 MTHF) to 5-methyltetrahydrofolate (5-MTHF). The former acts as a cofactor to form a stable complex with active metabolite of 5-FU i.e. fluoro-deoxy uridine monophosphate (FdUMP). Formation of the stable complex is required for effective inhibition of Thymidylate synthase enzyme (TS). MTHFR ${ }^{*} 4$ and $\mathrm{MTHFR}^{\star} 6$ are the most commonly reported SNP's in the MTHFR gene and are associated with altered enzyme activity and elevated levels of 5, 10 MTHF. Elevated levels of 5, 10 MTHF are required for the optimal effect of 5-FU and capecitabine.

A study by Thomas F in order to find the relationship between the SNP's in MTHFR gene with toxicity, tumor response and overall survival with $5 \mathrm{FU}$ therapy, reported a protective effect against toxicities like severe diarrhea and mucositis and no association with response and overall survival in the presence of these two polymorphisms [12].
In another study by Etienne et al. found that tumors cells having MTHFR polymorphisms (MTHFR ${ }^{\star} 4 \& \mathrm{MTHFR}^{\star} 6$ ) are more sensitive to 5-FU therapy but clinical data is not in line with experimental data and authors anticipated these difference may be due to interpatient variability in the folate status (Figure 1) [13].

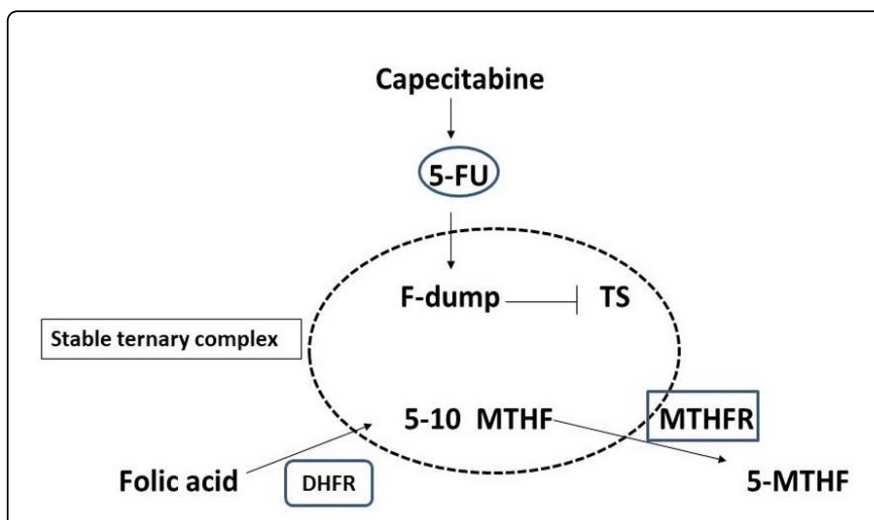

Figure 1: This figures the shows the formation of the stable ternary complex (F-dump+5-10 MTHF+TS) which is required for the effective inhibition of TS Enzyme. DHFR=Dihyrofolate reductase, MTHFR $=$ methylene tetrahydrofolate reductase, 5-10 MTHF $=5-10$ methylene tetrahydrofolate, $5 \mathrm{MTHF}=5$ methyl tetrahydrofolate.

Carboxylesterase (CES): CES exists in two isoforms, CES-1 and CES-2. CES-1 is predominantly expressed in liver and CES-2 in the small intestine. Many prodrugs like candesartan, aspirin, clopidogrel, azilsartan, irinotecan and including capecitabine depend on CES-2 mediated hydrolysis for their activation. Any alterations in the hydrolytic activity of CES-2 found to alter the therapeutic efficacy or toxicity of capecitabine [14].

A study conducted by Ribelles et al. for the first time found an association between genetic polymorphisms in CES-2 gene and efficacy of capecitabine. The study proved genetic variations CES-2 gene has predictive and prognostic value. 5'UTR 823 C-G polymorphism in CES-2 gene is found to alter the enzyme activity. Patients who were heterozygous and homozygous to this variation showed a significantly greater response $(59 \%)$ and time to disease progression ( 8.7 months) to capecitabine therapy compared to patients with wild-type allele (32\% and 5.3 months). The presence of another polymorphism, G-A on exon 3 at 6046 positions of CES 2 gene is associated with toxicities like grade 3 HFS and grade 3 and 4 diarrhea with capecitabine treatment in advanced breast cancer and metastatic colorectal cancer patients [15].

Cytidine deaminase (CDA): Nucleoside analogue produgs like gemcitabine, cytarabine, capecitabine and azacytidine are activated through CDA dependent metabolism. CDA converts intermediate metabolite of capecitabine i.e. 5-deoxy-5-fluoro cytidine to 5-deoxy-5fluoro uridine, which later acts as a precursor for the release of 5-FU. It was found that variations in CDA gene have been related to capecitabine toxicity.

A study by Caronia et al. genotyped 130 breast and colorectal cancer patients and found that two variants in the promoter of CDA gene rs532545 (-451 C>T) \& rs3215400 (-31 del C) are in linkage disequilibrium are strongly associated with the development capecitabine induced HFS [16]. In another study conducted by Gracia 
et al. in 239 colorectal cancer patients, it was found that the presence of $\mathrm{CDA} 79 \mathrm{~A}>\mathrm{C}$ variation was linked to overall toxicity to capecitabine therapy and observed a trend toward severe diarrhea [17].

Thymidine Phosphorylase (TP): TP is highly expressed in tumor cells and the key enzyme for the generation of 5-FU from capecitabine in tumor cells. It is considered to be a rate-limiting enzyme and play a key role in the efficacy of capecitabine. Higher expression of TP in tumor cells was found to increase the response rate with capecitabinebased chemotherapy and also TP overexpression is significantly associated with severe toxicities like HFS [18]. In contrast to this TP also proved to have a role in tumor neoangiogenesis, extracellular matrix remodelling, and metastasis. So it is very complex to rely on it as a marker for predicting the clinical response to capecitabine therapy [19].

A randomized noninferiority phase II trial with a pharmacogenetic analysis in 195 metastatic breast cancer patients found that the presence of two polymorphisms in TP gene (rs 11479 and rs131804) found to increase the overall survival (OS) with capecitabine treatment (Figure 2) [20].

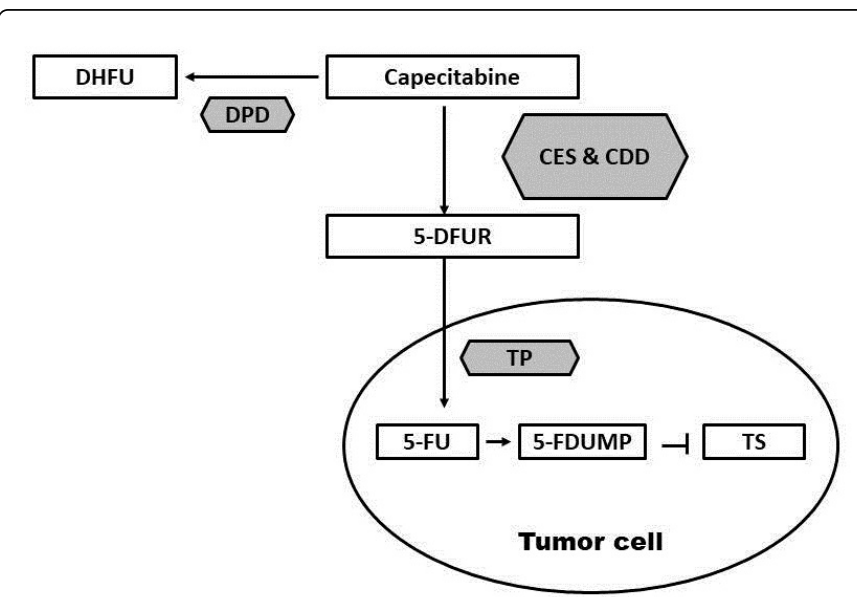

Figure 2: The figure shows different enzymes involved in activation capecitabine to 5-Flurouracil and its metabolism. $\mathrm{CES}=$ Carboxylesterase, $\quad \mathrm{CDD}=$ cytidine deaminase, 5 -DFUR $=5$ deoxy flurouridine, $\mathrm{TP}=$ thymidine phosphorylase, $5-\mathrm{FU}=5$ fluorouracil, 5-Fdump $=5$ flurodeoxyuridine monophosphate, TS=Thymidylate synthase, $\quad \mathrm{DPD}=$ dihydropyrimidine dehydrogenase, DHFU=Dihydroflurouracil.

Glutathione S-transferase (GST): Oxaliplatin is detoxified in the body through glutathione conjugation (GSH) reaction mediated by glutathione S-transferase enzyme (GST) and later excreted out through kidneys. The three major subtypes of GST are GST mu $(\mu)$, GST beta $(\beta)$ and GST-P (pi). Out of these three subtypes, GST-P is mainly involved detoxification of oxaliplatin. It was found that higher expression of GST-P enzyme causes drug resistance and whereas decreased activity is associated with severe toxicities like peripheral neuropathy [21].

A single nucleotide polymorphism of GSTP1 i.e. Ile105val (rs1695) is the most identified variation, which is associated with decreased enzyme activity. It was found that oxaliplatin-related cumulative neuropathy was significantly more frequent in patients carrying homozygous GSTP1 Ile105val allele (rs1695) variation [22].
Supporting these findings a study by Qing-Fang L et al. also found similar findings, the homozygous carriers Ile105val allele are at a higher risk of developing peripheral neuropathy with oxaliplatin-based chemotherapy [23].

UDP-glucuronosyl transferase-1A (UGT-1A): UGT-1A is an enzyme of glucuronidation pathway and catalyzes the transfer of glucuronic acid component to lipophilic drugs to make them hydrophilic and promote the easy excretion from the body. The active metabolite of irinotecan SN-38 depends on UGT-1A mediated conjugation reaction for elimination.

A meta-analysis suggests that the presence of a UGT1A1 ${ }^{\star} 28$ polymorphism in the TATA box is associated with decreased enzyme activity and it was found that individuals with this polymorphism are at a higher risk and develop severe neutropenia with irinotecan compared to normal individuals [24].

The FDA approved drug label also warns that the homozygous carries of $\mathrm{UGT} \mathrm{A} 1^{\star} 28$ polymorphism are at increased risk for developing severe neutropenia followed by irinotecan injection and recommends for dose reduction by 1 level in homozygous UGT1A1 ${ }^{\star} 28$ allele patients. However, the precise dose reduction is not known and those adjustments should be based on the individual tolerance to treatment. No dose reduction is recommended for heterozygous carriers with UGT1A1 ${ }^{\star 2} 8$ polymorphism [25].

\section{Drug targets}

Thymidylate synthase (TS): TS is a principal target for drugs like 5FU and capecitabine. Higher expression of TS in tumor cells is found to be associated with resistance to 5-FU treatment in colorectal cancer [26]. However, low TS expression does not necessarily predict higher response because the non-responders may have other mechanisms of drug resistance like elevated levels dihydropyrimidine dehydrogenase (DPYD). In addition to this few studies have linked genetic polymorphisms in thymidylate synthase gene (TYMS) with the prognosis of CRC [27]. However, a meta-analysis reported no such association between TS polymorphism and risk of CRC [28].

TYMS is found to be polymorphic in the promoter region, having either two repeats $(2 \mathrm{R})$ or three repeats (3R) of 28 base pairs, with three common genotypes, $2 \mathrm{R} / 2 \mathrm{R}, 2 \mathrm{R} / 3 \mathrm{R}$, and $3 \mathrm{R} / 3 \mathrm{R}$. The homozygous triple tandem repeat genotype $(3 R / 3 R)$ is associated with 3 fold increase in TS expression in tumor cells compared to double tandem repeats $(2 \mathrm{R} / 2 \mathrm{R})$.

A study by Pullarkat et al. found that patients with $2 \mathrm{R} / 2 \mathrm{R}$ had a response rate of $50 \%$ compared $3 \mathrm{R} / 3 \mathrm{R}$ genotype with $9 \%$ response rate and patients with the triple tandem $(3 \mathrm{R} / 3 \mathrm{R})$ genotype reported lesser adverse effects. The study suggested that genotyping of TYMS gene polymorphism have a potential to identify patients who are more likely to respond to 5-FU-based chemotherapy [29].

Topoisomerase-1 (Topo-1): Topoisomerase (Topo-1) enzyme promotes DNA replication by relaxing the supercoiled or torsional double stranded DNA. The active form of irinotecan, SN 38 exerts its antitumor activity by inhibiting topo-1. It was found that presence of mutated topo-1 enzyme has reduced affinity for irinotecan and causes drug resistance [30].

A randomized trial (FOCUS) accessed candidate predictive biomarkers for irinotecan and oxaliplatin in 1, 628 patients and reported genetic and epigenetic influence on Topo-1 expression levels. The trial reported the efficacy of irinotecan or oxaliplatin is dependent 
on the levels of the Topo-1 enzyme. In patients with low topo-1 levels, the progression-free survival was not improved either by adding irinotecan or oxaliplatin conversely and in patients with moderate or high levels of Topo-1 is benefited on adding of either drug [31].

A study by Paolicchi et al. analyzed for the association between two genetic polymorphisms (rs6072249 and rs34282819) in the promoter region of Topo-1 gene with clinical outcomes with irinotecan in metastatic CRC patients. No single genotype was found to predict the clinical outcomes (response rate, progression-free survival and overall survival). Further, well planned pharmacogenetic studies are required to detect predictive markers to irinotecan therapy [32].

Epidermal growth factor receptor (EGFR): Cetuximab and panitumumab are EGFR inhibitors approved in 2006 by US FDA. Both these drugs are add-on drugs for the management of CRC. Epidermal growth factor receptor (EGFR) is membrane bound tyrosine kinase receptor which plays a crucial role in cell proliferation and differentiation. Activated EGFR by ligand undergoes dimerization and further activate an intracellular transduction signalling molecule called KRAS. Activated KRAS further activates several other protein kinases like BRAF, MEK and ERK, which are required for cell multiplication. It was found that mutations in KRAS gene confer resistance to EGFR inhibitors [33]. KRAS gene mutations also found to cause abnormal cancerous growth and approximately $30-50 \%$ of colorectal cancers are known to have KRAS mutation [34].

Exon 2, $\mathrm{G} \rightarrow \mathrm{A}$ and $\mathrm{G} \rightarrow \mathrm{T}$ transitions on codons 12 and 13 respectively and to a lesser extent an EXON 3, G $\rightarrow$ A transition on codon 61 are the most well-established variations of KRAS gene. The presence of these KRAS mutations was found to be associated with the less or no clinical effectiveness of EGFR inhibitors [35].

In 2009 US FDA updated the product label of panitumumab and cetuximab indicating that the presence of KRAS mutations is associated with lack of benefit with epidermal growth factor inhibitors [36]. In 2012, FDA approved a PCR companion diagnostic genetic test for detecting the presence of KRAS gene mutations. The positive screening results of these mutations indicate that the patients may not benefit from the treatment with EGFR inhibitors (Figure 3) [37].

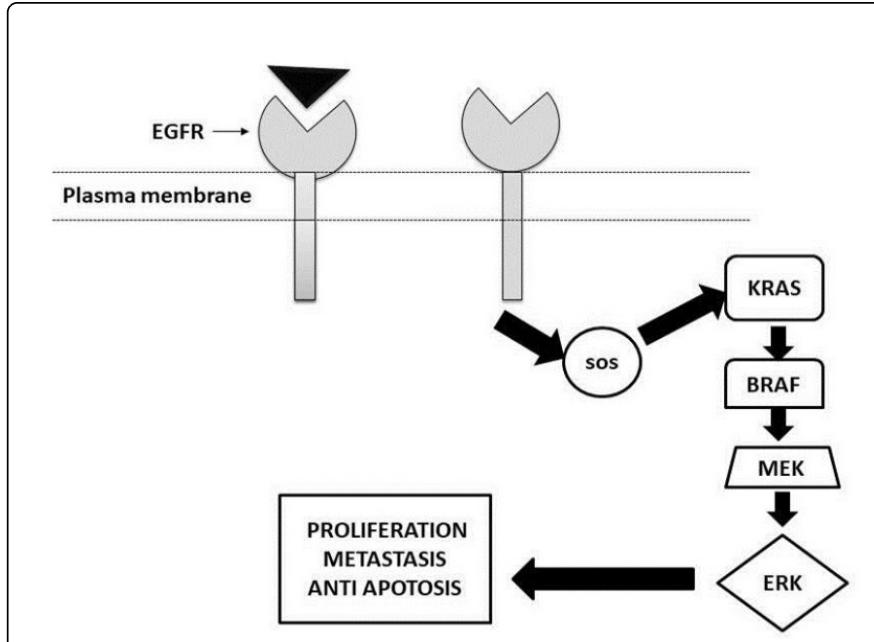

Figure 3: This figure shows EGFR activation and the downstream signal transduction leading to cell proliferation and metastasis. $\mathrm{EGFR}=$ epidermal growth factor receptor, $\mathrm{SOS}=$ son of sevenless factor, KRAS=Kirsten rat sarcoma virus GTPase, $\mathrm{BRAF}=\mathrm{Raf}$ murine sarcoma viral oncogene homolog $\mathrm{B}, \mathrm{MEK}=$ mitogen activated protein kinases, ERK=Extra cellular signal regulator kinases.

Vascular endothelial growth factor: Bevacizumab is a vascular endothelial growth factor- A (VEGF-A) inhibitor, approved in 2014 by US FDA. VEGF-A is an angiogenic factor and it plays an important role in endothelial cell growth and neovascularization for the growing tumors. VEGF-A expression levels are generally considered to be one of the important markers for tumor progression and invasiveness. It was found that overexpression of VEGF-A is usually associated with a poor survival rate in CRC patients and causes resistance to bevacizumab treatment in metastatic CRC patients [38].

Meta-analysis on VEGF gene polymorphisms exclusively reported about 5 SNP's i.e. $+936 \mathrm{C}>\mathrm{T},-460 \mathrm{~T}>\mathrm{C},+405 \mathrm{G}>\mathrm{C},-1154 \mathrm{G}>\mathrm{A}$, and $-2578 \mathrm{C}>\mathrm{A}$. The presence of these polymorphisms is associated to have a better prognosis with anti-VEGF therapy. Out of these 5 polymorphisms, the presence of $+405 \mathrm{G}>\mathrm{C}$ variant showed high statistical significance in the improvement of overall survival [38].

A phase III randomized clinical study by Schneider et al. also found similar findings, the presence of two variants (VEGF-A -2578 C/A and VEGF - $1154 \mathrm{G} / \mathrm{A}$ ) were independently associated with a superior median overall survival in metastatic breast cancer patients treated with paclitaxel +bevacizumab [39].

\section{DNA damage repair}

Excision Repair Cross-Complementation (ERCC): Drugs like oxaliplatin inhibits the cancer cell proliferation by forming DNA adducts or cross links. The ability of cancer cell to repair damaged DNA is one of the known mechanisms for drug resistance. Nucleotide excision repair pathway (NER) is a major repair pathway involved in removing DNA cross-links. Two important and rate-limiting proteins of NER pathway are excision repair cross-complementation 1 (ercc1) and xeroderma pigmentosum group A (XPA) [40].

High Expression of ERCC proteins in tumor cells was found to be associated with resistance to oxaliplatin treatment while relatively low levels of these protein have been associated with higher sensitivity [41]. 
Citation: Varma A, Jayanthi M and Shewade DG (2018) Single Nucleotide Polymorphisms as Biomarkers for Drug Response and Toxicity in the Management of Colorectal Cancer. J Pharmacogenomics Pharmacoproteomics 9: 178. doi:10.4172/2153-0645.1000178

Page 5 of 6

A meta-analysis based on several studies reported, the presence of two polymorphisms in ERCC pathway i.e. ERCC1 ( $r s 11615 \mathrm{C}>\mathrm{T}$ ) and ERCC2 (rs13181T $>$ G) are associated with poor response, PFS and OS rates in gastric and metastatic colorectal cancer patients treated with oxaliplatin-based chemotherapy [42]

A synonymous $(\mathrm{C} \rightarrow \mathrm{T})$ variation in codon 118 of ERCC1 was also found to cause increased expression of the ERCC1 protein and associated with poor response and prognosis with oxaliplatin-based therapy (Table 2) [43].

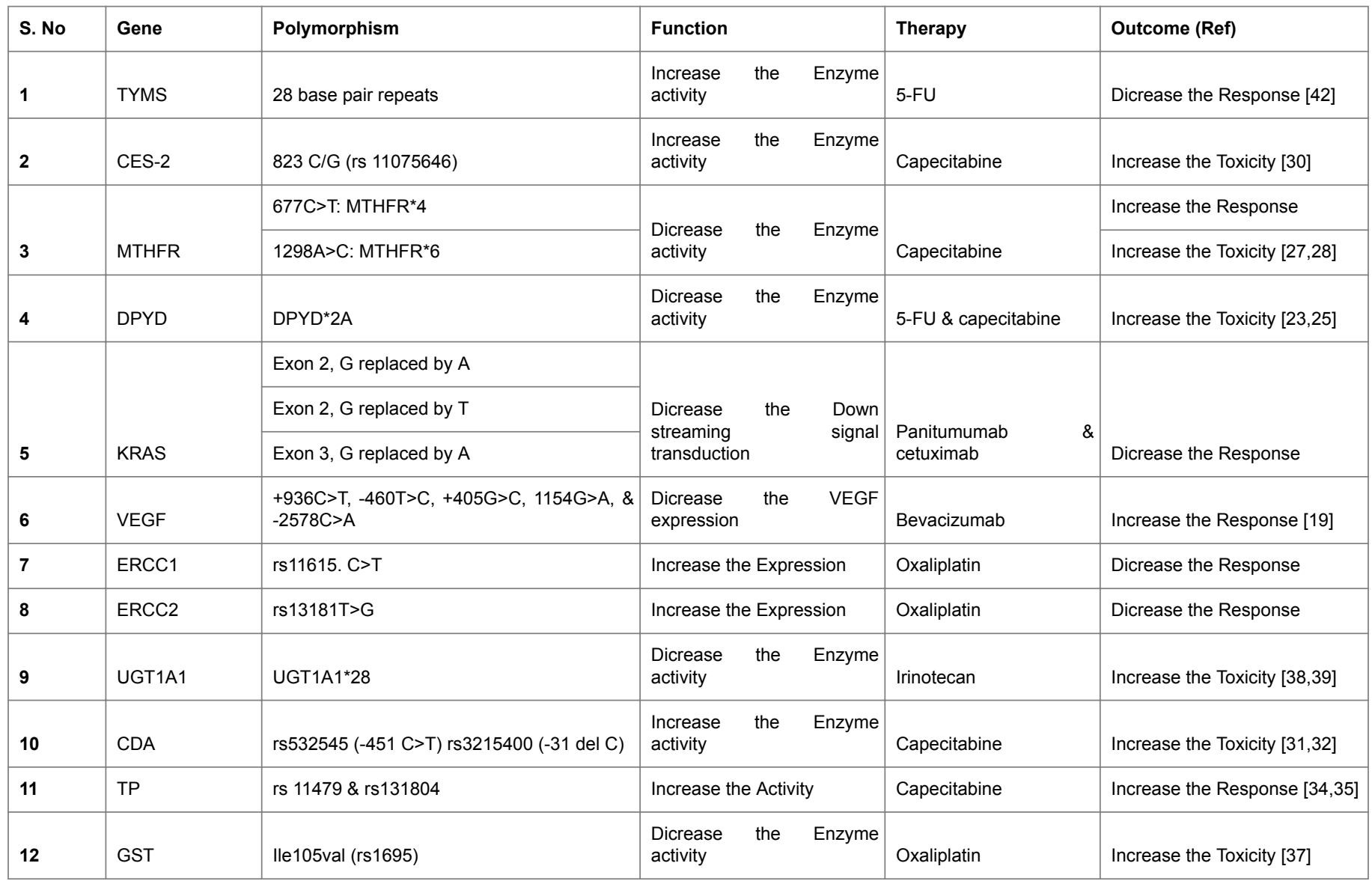

Table 2: Predictive markers in colorectal cancer in a nutshell.

\section{Conclusion}

Identifying the predictive biomarkers for treatment efficacy and safety in CRC management is a difficult task because patients are usually treated with combinational therapy and many factors like disease stage, concomitant diseases, age, sex etc. can influence the drug response status. The individual genetic makeup especially genetic polymorphisms in the drug metabolizing enzymes, drug targets and DNA repair proteins are exclusively studied to identify specific predictive markers to drug response. Few of these genetic markers are successful in predicting the optimal drug and dose for the patients. However it is very complex to identify the single nucleotide polymorphisms that are to known affect the drug response and to use them as predictive markers in the routine clinical practice because of cost involved and ethnicity variations. In this respect, prospective clinical trials related to CRC patients should aim to find the effective predictive markers and help to develop easy-to-implement tools to design individualize dosing based upon the patient's genotypes.

\section{References}

1. Cappell MS (2008) Pathophysiology, Clinical Presentation, and Management of Colon Cancer. Gastroenterol Clin North Am 37: 1-24.

2. Haggar FA, Boushey RP (2009) Colorectal Cancer Epidemiology: Incidence, Mortality, Survival, and Risk Factors. Clin Colon Rectal Surg 22: 191-197.

3. Sirohi B, Shrikhande SV, Perakath B, Raghunandharao D, Julka PK, et al. (2014) Indian Council of Medical Research consensus document for the management of colorectal cancer. J Indian Soc Med Paediatr Oncol 35: 1926.

4. Moghimi-Dehkordi B, Safaee A (2012) An overview of colorectal cancer survival rates and prognosis in Asia. World J Gastrointest Oncol 4: 71-75.

5. Walther A, Johnstone E, Swanton C, Midgley R, Tomlinson I, et al. (2009) Genetic prognostic and predictive markers in colorectal cancer. Nat Rev Cancer 9: 489-499.

6. Del Re M, Loupakis F, Barbara C, Lombardo L, Latiano T, et al. (2014) A clinical pharmacogenetic characterization of DPD polymorphisms for pre-treatment screening of patients candidates to fluoropyrimidine therapy. EPMA J 5: S1-A24 
7. Li Q, Liu Y, Zhang H-M, Huang Y-P, Wang T-Y, et al. (2014) Influence of DPYD Genetic Polymorphisms on 5-Fluorouracil Toxicities in Patients with Colorectal Cancer: A Meta-Analysis. Gastroenterol Res Pract 2014.

8. Dean L (2016) Fluorouracil Therapy and DPYD Genotype - Medical Genetics Summaries - NCBI Bookshelf.

9. Surjushe A, Vasani R, Medhekar S, Thakre M, Saple DG (2009) Handfoot syndrome due to capecitabine. Indian J Dermatol 54: 301.

10. Borro M, Botticelli A, Mazzuca F, Onesti EC, Gentile G, et al. (2016) Pretreatment assay of 5-fluorouracil degradation rate (5-FUDR) to improve prediction of 5-fluorouracil toxicity in gastro-esophageal cancer. Oncotarget 8: 14050-14057.

11. Gentile G, Botticelli A, Lionetto L, Mazzuca F, Simmaco M, et al. (2016) Genotype-phenotype correlations in 5-fluorouracil metabolism: a candidate DPYD haplotype to improve toxicity prediction. Pharmacogenomics J 16: 320-325.

12. Thomas F, Motsinger-Reif AA, Hoskins JM, Dvorak A, Roy S, et al. (2011) Methylenetetrahydrofolate reductase genetic polymorphisms and toxicity to 5-FU-based chemoradiation in rectal cancer. Br J Cancer 105: 1654-1662.

13. Etienne-Grimaldi M-C, Francoual M, Formento J-L, Milano G (2007) (Methylenetetrahydrofolate reductase (MTHFR) variants and fluorouracil-based treatments in colorectal cancer. Pharmacogenomics 8: 1561-1566.

14. Laizure SC, Herring V, Hu Z, Witbrodt K, Parker RB (2013) The role of human carboxylesterases in drug metabolism: have we overlooked their importance? Pharmacotherapy 3: 210-222.

15. Ribelles N, López-Siles J, Sánchez A, González E, Sánchez MJ, et al. (2008) A carboxylesterase 2 gene polymorphism as predictor of capecitabine on response and time to progression. Curr Drug Metab 9: 336-343.

16. Caronia D, Martin M, Sastre J, de la Torre J, García-Sáenz JA, et al. (2011) A polymorphism in the cytidine deaminase promoter predicts severe capecitabine-induced hand-foot syndrome. J Am Assoc Cancer Res 17: 2006-2013.

17. García-González X, Cortejoso L, García MI, García-Alfonso P, Robles L, et al. (2015) Variants in CDA and ABCB1 are predictors of capecitabinerelated adverse reactions in colorectal cancer. Oncotarget 6: 6422-6430.

18. Han J-Y, Hong EK, Lee SY, Yoon SM, Lee DH, et al. (2005) Thymidine phosphorylase expression in tumour cells and tumour response to capecitabine plus docetaxel chemotherapy in non-small cell lung cancer. J Clin Pathol 58: 650-654.

19. Bronckaers A, Gago F, Balzarini J, Liekens S (2009) The dual role of thymidine phosphorylase in cancer development and chemotherapy. Med Res Rev 29: 903-953.

20. Martín M, Martínez N, Ramos M, Calvo L, Lluch A, et al. (2015) Standard versus continuous administration of capecitabine in metastatic breast cancer (GEICAM/2009-05): a randomized, noninferiority phase II trial with a pharmacogenetic analysis. The Oncologist 20: 111-112.

21. Noda E, Maeda K, Inoue T, Fukunaga S, Nagahara H, et al. (2012) Predictive value of expression of ERCC 1 and GST-p for 5-fluorouracil/ oxaliplatin chemotherapy in advanced colorectal cancer Hepatogastroenterology 59: 130-133.

22. Lecomte T, Landi B, Beaune P, Laurent-Puig P, Loriot MA, et al. (2006) P1 polymorphism (ILe105Val) predicts cumulative neuropathy in patients receiving oxaliplatin-based chemotherapy. Clin Cancer Res 12: 3050-3056

23. Li Q-F, Yao R-Y, Liu K, Lv H-Y, Jiang T, et al. (2010) Genetic Polymorphism of GSTP1: Prediction of Clinical Outcome to Oxaliplatin/5-FU-based Chemotherapy in Advanced Gastric Cancer. J Korean Med Sci 25: 846-852.

24. Dias MM, McKinnon RA, Sorich MJ (2012) Impact of the UGT1A1*28 allele on response to irinotecan: a systematic review and meta-analysis. Pharmacogenomics 13: 889-899.
25. Dean L (2012) Irinotecan Therapy and UGT1A1 Genotype. Medical Genetics Summaries National Center for Biotechnology Information (US).

26. Leichman CG, Lenz HJ, Leichman L, Danenberg K, Baranda J, et al. (1997) Quantitation of intratumoral thymidylate synthase expression predicts for disseminated colorectal cancer response and resistance to protracted-infusion fluorouracil and weekly leucovorin. J Am Soc Clin Oncol 15: 3223-3229.

27. Mirjolet JF, Barberi-Heyob M, Merlin JL, Marchal S, Etienne MC, et al. (1998) Thymidylate synthase expression and activity: relation to S-phase parameters and 5-fluorouracil sensitivity. Br J Cancer 78: 62-68.

28. Popat S, Matakidou A, Houlston RS (2004) Thymidylate Synthase Expression and Prognosis in Colorectal Cancer: A Systematic Review and Meta-Analysis. J Clin Oncol 22: 529-536.

29. Pullarkat ST, Stoehlmacher J, Ghaderi V, Xiong YP, Ingles SA, et al. (2001) Thymidylate synthase gene polymorphism determines response and toxicity of 5-FU chemotherapy. The Pharmacogenomics J 10: 65-70.

30. Gongora C, Vezzio-Vie N, Tuduri S, Denis V, Causse A, et al. (2011) New Topoisomerase I mutations are associated with resistance to camptothecin. Mol Cancer 10: 10-64.

31. Braun MS, Richman SD, Quirke P, Daly C, Adlard JW, et al. (2008) Predictive biomarkers of chemotherapy efficacy in colorectal cancer: results from the UK MRC FOCUS trial. J Am Soc Clin Oncol 26: 2690-2698.

32. Paolicchi E, Vivaldi C, De Gregorio V, Crea F, Fornaro L, et al. (2016) Topoisomerase 1 Promoter Variants and Benefit from Irinotecan in Metastatic Colorectal Cancer Patients. Oncology 91: 283-288.

33. Raponi M, Winkler H, Dracopoli NC (2008) KRAS mutations predict response to EGFR inhibitors. Curr Opin Pharmacol 8: 413-418.

34. Tan C, Du X (2012) KRAS mutation testing in metastatic colorectal cancer. World J Gastroenterol WJG 18: 5171-5180.

35. Arrington AK, Heinrich EL, Lee W, Duldulao M, Patel S, et al. (2012) Prognostic and Predictive Roles of KRAS Mutation in Colorectal Cancer. Int J Mol Sci. 13: 12153-12168.

36. Shankaran V, Obel J, Benson AB (2010) Predicting Response to EGFR Inhibitors in Metastatic Colorectal Cancer: Current Practice and Future Directions. The Oncologist 1: 157-167.

37. FDA Approves Genetic Test for KRAS Mutation in CRC Patients. Medscape.

38. Eng L, Azad AK, Habbous S, Pang V, Xu W, et al. (2012) Vascular endothelial growth factor pathway polymorphisms as prognostic and pharmacogenetic factors in cancer: a systematic review and metaanalysis. J Am Assoc Cancer Res 18: 4526-4537.

39. Schneider BP, Wang M, Radovich M, Sledge GW, Badve S, et al. (2008) Association of vascular endothelial growth factor and vascular endothelial growth factor receptor-2 genetic polymorphisms with outcome in a trial of paclitaxel compared with paclitaxel. J Clin Oncol 26: $4672-4678$.

40. Reed E (2005) ERCC1 and clinical resistance to platinum-based therapy. J Am Assoc Cancer Res 11: 6100-6102.

41. Strippoli A, Basso M, Orlandi A, Schinzari G, Calegari A, et al. (2012) New biomarkers to predict response to oxaliplatin-based chemotherapy in metastatic colorectal cancer: KRAS and ERCC1. J Clin Oncol 30: 500

42. Yin M, Yan J, Martinez-Balibrea E, Graziano F, Lenz H-J, et al (2011) ERCC1 and ERCC2 polymorphisms predict clinical outcomes of oxaliplatin-based chemotherapies in gastric and colorectal cancer: a systemic review and meta-analysis. J Am Assoc Cancer Res 17: 1632-1640.

43. Viguier J, Boige V, Miquel C, Pocard M, Giraudeau B, et al. (2005) ERCC1 codon 118 polymorphism is a predictive factor for the tumor response to oxaliplatin/5-fluorouracil combination chemotherapy in patients with advanced colorectal cancer. J Am Assoc Cancer Res 11: 6212-6217. 